\title{
Networks of Interruptions: Simultaneous Speech and Leader Emergence in Informal Groups
}

Neil G. MacLaren ${ }^{1}$, Francis J. Yammarino ${ }^{1}$, Shelley D. Dionne ${ }^{1}$, Hiroki Sayama ${ }^{1}$, Michael D. Mumford ${ }^{2}$, Shane Connelly ${ }^{2}$, Robert W. Martin ${ }^{2}$, Colleen J. Standish ${ }^{2}$, Samantha England ${ }^{2}$, Tanner R. Newbold ${ }^{2}$, Yiding Cao ${ }^{1}$, Jason Marshall ${ }^{1}$, Shun Cao ${ }^{1}$, Yingjun Dong ${ }^{1}$, Cynthia K. Maupin ${ }^{1}$, Rory Eckardt ${ }^{1}$, and Gregory A. Ruark ${ }^{3}$

${ }^{1}$ Binghamton University, State University of New York, ${ }^{2}$ University of Oklahoma, ${ }^{3}$ United States Army Research Institute for the Social and Behavioral Sciences

June 30, 2021

\section{Author Note}

The research described herein was sponsored by the U.S. Army Research Institute for the Behavioral and Social Sciences, Department of the Army (Grant No. W911NF-17-1-0221). The views expressed in this manuscript are those of the authors and do not reflect the official policy or position of the Department of the Army, DOD, or the U.S. Government.

Corresponding author is Neil G. MacLaren, nmaclar1@binghamton.edu, School of Management, Binghamton University, PO Box 6000, Binghamton, NY 13902-6000, UNITED STATES.

The data used in this study is available at https://orb.binghamton.edu/management_fac/2/. Analysis code is located at https://github.com/ngmaclaren/interruption-networks. 


\begin{abstract}
There is a demonstrable association between the amount of time that a member of a small, informal group speaks and the probability that other group members will attribute emergent leader status to that individual. However, speaking time cannot represent the pattern of relationships in small groups that many theories of leadership propose. In this study, we derive interruptions and related behaviors from previously gathered records of who spoke when in 33 groups, then test whether networks of these behaviors predict social status over and above the effect of speaking time. Two-stage least squares analysis, simulation studies, and exponential random graph analysis suggest that interruptions may be associated with status alongside speaking time, and further that network analysis of conversational behaviors provides a window into the interaction patterns and social structure of small groups.
\end{abstract}

Keywords: leader emergence; network analysis; nonverbal behavior; sociometric status; gender

\title{
Introduction
}

Social status varies between individuals in work groups, whether emergent or by design (Bendersky \& Pai, 2018). Classic studies have suggested that social hierarchy influences decision speed and accuracy (e.g., Bavelas, 1950), and that tension between a prescribed and emergent hierarchy results in detrimental outcomes on some tests and beneficial outcomes on others (Torrance, 1954). More recent studies have highlighted the effects of within-group status on what information is discovered (Stasser, Abele, \& Parsons, 2012) and used (Becker, Brackbill, \& Centola, 2017) in decision making. Importantly, social status and leadership are connected constructs with the potential for confounding and associated deleterious effects (Lanaj \& Hollenbeck, 2015). Understanding social status in the small group building blocks of organizations, then, has both theoretical and practical importance.

There is a growing recognition that understanding the patterns of observable interaction behaviors of group members may provide a window into the "black box" of group process (Gorman et al., 2020; Matusik et al., 2019; Riedl, Kim, Gupta, Malone, \& Woolley, 2021). Some behavioral predictors of within-group status are well-established, such as the relationship between speaking time, or the cumulative duration of speech activity associated with a given individual, and leader emergence (MacLaren et al., 2020). However, an emphasis on speaking time, useful as an identifier of a single emergent leader, may underdescribe the relationships between all interacting members of a group. Interaction behaviors that do potentially correspond to such relationships, such as conversational interruptions, are a potential solution, but the literature contains several conflicting findings (Hall, Coats, \& LeBeau, 2005) that undermine strong statements about the association between interruptions and social status (e.g., Smith-Lovin \& Brody, 1989).

There are two primary conflicting results in the literature on interruptions and social status. First, speaking time has long been known to confound studies of behavior in small groups (Bavelas, Hastorf, Gross, \& Kite, 1965), and it appears to do so for interruptions: the relationship between interruptions and assessed within-group status is greatly reduced in studies which directly control for speaking time (Dabbs, Ruback, \& Evans, 1987). 
Second, laboratory- and field-based studies yield differing perspectives, with some (Hall \& Friedman, 1999; Irish \& Hall, 1995) but not all (Feldman \& Gill, 2019) field studies finding that higher status individuals interrupt less, whereas both observational (Reynolds, 1984) and experimental (Farley, 2008) laboratory studies tend to associate higher social status with those who interrupt more.

In this study, we focus on the first conflict, the potential confounding effect of speaking time on the relationship between interruptions and assessed social status, and in so doing we develop a method that can be used to address the second, the discrepancy between laboratory- and field-based research. Our essential instight is that a network perspective on interaction behaviors both better represents the theory of social status (Berger, Cohen, \& Zelditch, 1972) and may better account for the importance of who behaves in what way to whom. We therefore pursue a "group parts" approach in a multi-level framework (Yammarino \& Gooty, 2019) that explicitly views individuals as having important dyadic relationships with other individuals as opposed to contributing to an undifferentiated group. Through the use of "interruption networks" we find that interruption behaviors positively predict assessed social status in informal groups, but not necessarily in a straightforward way-at least, not when accounting for speaking time: it's the pattern of who defers to whom that matters, as indicated by patterns in simultaneous speech.

Unraveling these relationships between behavior and perception has several potential benefits. From a psychophysical perspective, there are quesitons about what aspects of the information environment lead group members to make certain attributions-in this case, of relative social status. A more strictly behavioral perspective may simply ask, in reference to the "babble hypothesis" (MacLaren et al., 2020): what exactly do people do while they are babbling? A focus on how non-content features of speech relate to social status, although perhaps missing substantial nuance in communication content, is potentially more generalizable across task contexts, even languages (Heldner \& Edlund, 2010), because the pragmatic meaning of prosodic features of speech is not dependent on the semantic meaning of the words involved (Ward, 2019). Studying patterns of interruptions may lead to a greater understanding of the behavioral and relationship dynamics within work groups and contribute to leader and group assessment and training.

\section{Theory and Background}

The primary focus of this study is on behavioral indicators of social status. Social status refers to the "prestige, respect, and esteem that a party has in the eyes of others" (Bendersky \& Pai, 2018, p. 184): it is considered to be a fundamental human motive (Anderson, Hildreth, \& Howland, 2015), and those who possess it command deference and respect from those around them (Von Rueden \& Van Vugt, 2015). Members of small groups engage in a wide variety of communication behaviors related to social status, including aspects of posture, facial expression, proximity, vocal pitch, word choice-the list is neither exhaustive nor expected to be mutually exclusive (Anderson et al., 2015; Hall et al., 2005). Furthermore, group members and observers rapidly perceive a rich set of cues about the states, abilities, and relative social positions of relevant others (Gerpott, Lehmann-Willenbrock, Silvis, \& Van Vugt, 2018; Schmid Mast \& Hall, 2004).

Differentiation in status is also a common feature of work groups (Bendersky \& Pai, 2018; Krackhardt, 1994) that may or may not align well with organizational hierarchy (DeRue \& 
Ashford, 2010) or leader effectiveness (Lanaj \& Hollenbeck, 2015; Zaccaro, Green, Dubrow, \& Kolze, 2018).

Standard definitions of leadership accommodate the idea of deference implied by the terms status and prestige (Bass, 2008; Henrich, Chudek, \& Boyd, 2015). Indeed, common assessment procedures for leader emergence, social status, and dominance overlap substantially and share a common nomological network with observable behaviors (Hall et al., 2005; Schmid Mast, 2002) — a potential example of construct proliferation (Banks, Gooty, Ross, Williams, \& Harrington, 2018). Empirical evidence suggests both prestige and dominance behaviors lead to higher rankings of status (Cheng, Tracy, Foulsham, Kingstone, \& Henrich, 2013), consistent with a common dimension (Hall et al., 2005). Furthermore, some behaviors may be seen as unwanted, leading to reduced ratings of "liking" and similar constructs (Martinko et al., 2018; Yammarino, Cheong, Kim, \& Tsai, 2020), yet still result in increased ratings of status (Farley, 2008). Leaders or other individuals high in status may have positive, prosocial motivations or be manipulative and selfish (Alvesson \& Einola, 2019), yet other group members appear to rate such individuals in accordance with attributions of higher status - and potentially to defer to them behaviorally as well. Status and leadership are therefore not synonymous, but the study of each is conceptually and empirically entwined.

Researchers have argued for decades that social status is related to consequential outcomes for both the individual and the group (Anderson et al., 2015; Von Rueden \& Van Vugt, 2015). However, research on behavioral indicators of status has yielded mixed results. For example, the Hall et al. (2005) review indicates substantial differences in empirical relationships between status and several nonverbal behaviors when status is "perceived," or assessed by observers or fellow group members, or "actual," such as when status is organizationally defined. Some difficulty certainly resides in conflicting operationalizations (James \& Clarke, 1993), but additional difficulty lies in the substantial confound of participation amount (Bavelas et al., 1965; Gerpott et al., 2018), often assessed as speaking time (MacLaren et al., 2020).

\section{Simultaneous Speech}

Informally, an interruption occurs when one individual displaces another as the primary speaker in an interacting group. A related behavior, sometimes called an interjection or unsuccessful interruption, occurs when one individual starts speaking during another speaker's turn but does not displace the current speaker as the primary speaker. These behaviors are grouped together under the concept of simultaneous speech, and may be referred to as interruptive and non-interruptive simultaneous speech, or ISS and NSS, respectively (Feldstein \& Welkowitz, 1987). Both types of simultaneous speech may be important indicators of leader status. Reynolds (1984) found, in that study's terms, that leaders tend to "hold the floor" better than non-leaders, tending to both interrupt and resist interruption. Dabbs and Ruback (1987) report that participants rated higher in leadership by their fellow participants tended to display less non-interruptive simultaneous speech and more interruptive simultaneous speech. Importantly, ISS and NSS have qualitatively opposite interpretations-someone "yields the floor" to the focal group member in the former case, but the group member "yields" to someone else in the latter-yet both suggest that one individual has deferred to another, potentially indicating a source, target, and 
Figure 1: Interruptive and Non-Interruptive Simultaneous Speech
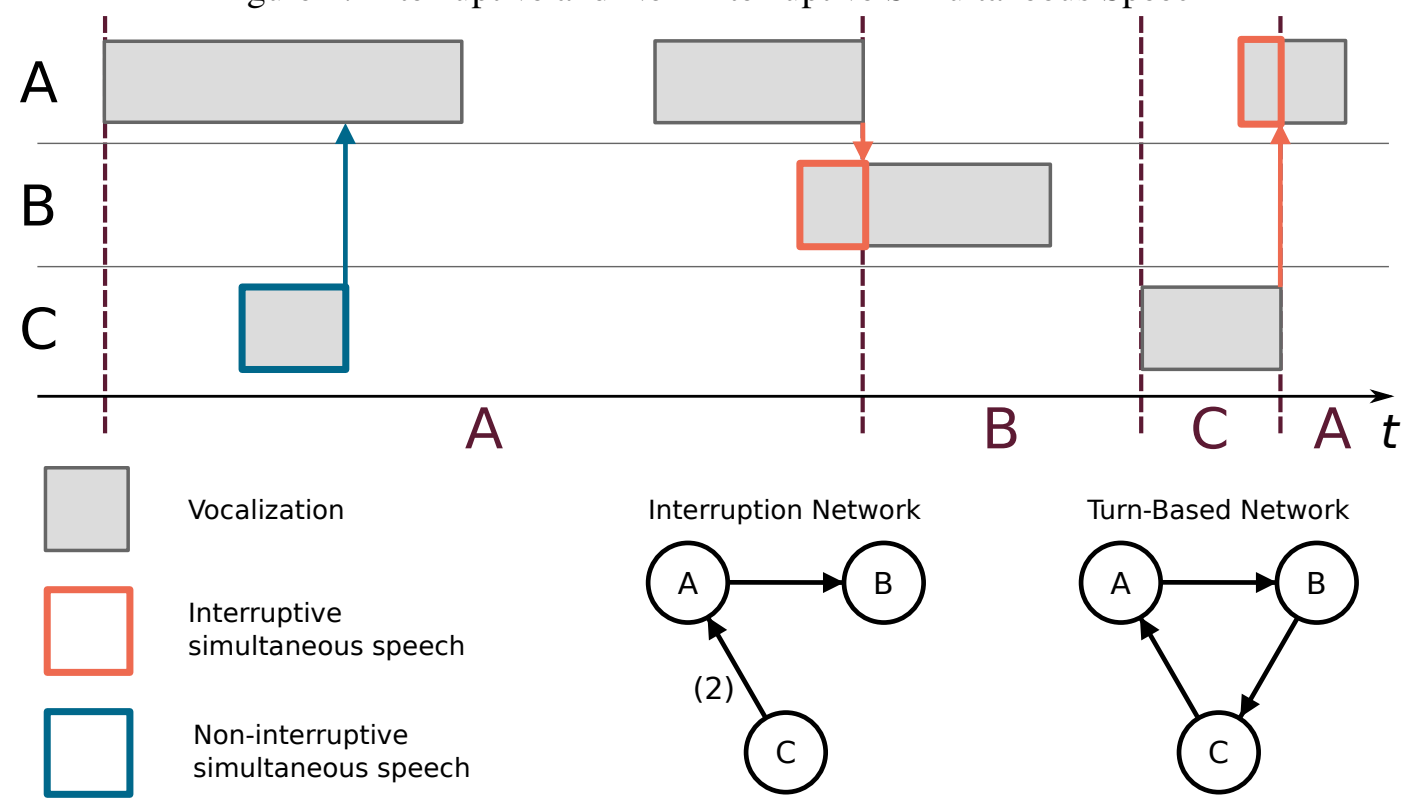

Note: A diagram of interruptive (red) and non-interruptive (blue) simultaneous speech and speaking turns (purple) in an example conversation. The minimal interruption (left) and turn-based (right) networks representing these interactions are shown in the lower right. In the interruption network, edges point towards the individual who "held the floor" at the end of the simultaneous speech event. If non-simultaneous speech is considered, then the edge from $\mathrm{C}$ to $\mathrm{A}$ would have a weight of two instead of one. In the turn-based network, edges point in sequential speaking turn order.

"flow" or "direction" in a status space.

Relying on diarized conversation data - that is, conversations for which the speaker, start time, and duration of speaking events have been recorded-Sanchez-Cortes, Aran, Jayagopi, Schmid Mast, and Gatica-Perez (2013, p. 45) use the following definition of an interruption, similar to that used in Tahir et al. (2019) and Farley (2008) and equivalent to interruptive simultaneous speech (ISS) as defined by Feldstein and Welkowitz (1987): "Participant A interrupts participant B if A starts talking when B is speaking; when A finishes his/her turn B is not speaking anymore" (Figure 1). Non-interruptive simultaneous speech can be expressed in similar language: a case in which A started speaking while B was speaking but A stopped speaking before B did. Importantly, these definitions allow a coder - human or machine - to focus only on identifying the start and end time of group member speaking events: simultaneous speech is assessed after the data is collected and coded. Although diarization by human or machine is not a trivial task, it is a task that requires relatively less interpretation than determining types of interruptions, which may be prone to bias or other error on the part of the coder (Hall, Horgan, \& Murphy, 2019).

A related concept is that of the speaking turn. Although the validity of the speaking turn construct has been called into doubt by recent studies (Laskowski, 2010; Ward, 2019), the construct is in common use and will be preserved here, at least as a useful analytical heuristic. A speaking turn "begins the instant one participant in a conversation starts talking 
alone and ends immediately prior to the instant another participant starts talking alone" (Feldstein \& Welkowitz, 1987, p. 441; Figure 1). Using the turn construct, ISS ends a speaking turn and begins another whereas NSS does not.

\section{A Network Perspective}

As others have pointed out (e.g., Smith-Lovin \& Brody, 1989), there is a mechanistic connection between speaking time, speaking turns, and simultaneous speech: one must speak to interrupt, speech is broken into turns by definition, and the more one speaks the more opportunities one has to exhibit speech behaviors of any kind, including simultaneous speech. However, both simultaneous speech and the flow of speaking turns suggest clear direction in behavior (Feldstein \& Welkowitz, 1987; Sauer \& Kauffeld, 2013): one person interrupts another, one person's turn follows another's. Such data may be analyzed as a network.

It should be pointed out here that the most common network paradigm in leadership research is the study of habitual relationships (see Carter, DeChurch, Braun, \& Contractor, 2015 , for a review). Rather than borrowing from theory derived from such studies, the present effort is more about determining what relationships may exist between observable behaviors and these habitual relationships; we are interested in the microfoundations of social status in groups. Network analysis is notable for its suite of analytical tools that so easily cross disciplinary boundaries (Sayama, Cramer, Porter, Sheetz, \& Uzzo, 2016), and in this study we use these tools without necessarily refering to sociological theory associated with them. Instead, the idea is more to understand the connections within groups - to go beyond the idea of individuals contributing to an undifferentiated "whole" group to look at the "group parts" perspective (Yammarino \& Gooty, 2019) from a multilayer (or multiplex) network perspective (Kivelä et al., 2014). A representation of a single group of participants in several network layers, explained in detail below, and including the speech data from which several layers are drawn, can be found in Figure 2.

\section{Vote Networks}

Carter et al. (2015) reviewed a variety of network studies with bearing on teams and/or leadership research: what they called "leadership networks" often consist of directed leadership attributions between members of a group, and could represent either leadership "claiming", "granting", or both; often, but not always, these are assessed with a survery measure, name generator, or similar. Although leader emergence studies and leadership network studies rely on similar survey assessment tools, leadership network studies are specifically interested in who attributed what to whom-the network aspect of the study. We will call this type of network, which we consider one layer in a multilayer network representation of a group, a "vote network" to distinguish is from other networks or network layers that may describe the same group and contain information relevant to leadership. In vote networks, we draw an edge from $i$ to $j$ if $i$ nominated $j$ as an emergent leader. Vote networks also provide another way to see connections between the leader emergence literature and studies of social status: a kind of importance in such networks, in-degree centrality, or the count of edges that point towards a node representing an individual on a network, is exactly equivalent to a count of nominations of an individual as an emergent leader. 
Figure 2: Example Networks
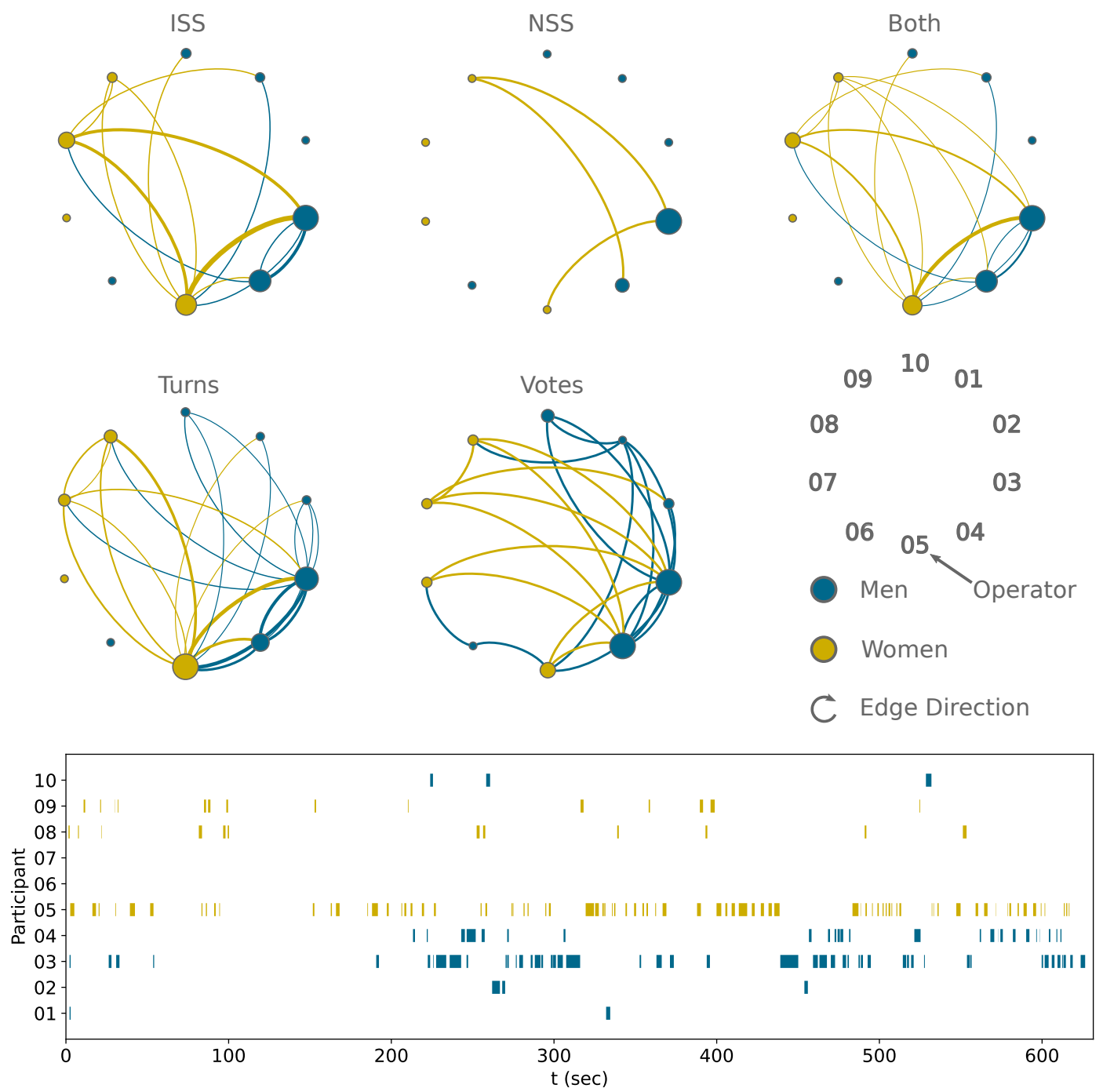

Note: Diarized speaking data from an example group (bottom) and several different network representations of the focal group: interruption networks using interruptive simultaneous speech only (ISS, top left), non-interruptive simultaneous speech (NSS, top middle), and both interruptive and non-interruptive simultaneous speech (Both, top right); a network based on speaking turns (middle left), and a network based on leader emergence votes (center). Men are marked in blue and women in gold throughout; the group's randomly assigned operator is participant "05;" and edges curve clockwise from source to target. Filled bars in the bottom panel indicate periods in which that participant was speaking. Individuals are represented by nodes in the same order in each network drawing. The size of each node is proportional to centrality in each network layer, and the thickness of an edge corresponds to its weight. 


\section{Turn-Based Networks}

Networks based on conversational turns were proposed by Sauer and Kauffeld (2013). To represent conversational turns in a network, an edge is drawn from $i$ to $j$ if $j$ 's turn follows $i$ 's; the edge $i \rightarrow j$ has a weight equal to the number of times $j$ immediately followed $i$ as the primary speaker (Figure 1). If one individual tends to speak after another, an edge will be drawn from the latter individual's node to the former's, potentially associated with a large edge weight. Nodes will tend to be important in this type of network if the represented individual speaks frequently, in particular if that individual speaks after many other individuals. Sauer and Kauffeld (2013) proposed that weighted in-degree centrality - that is, the sum of weights on all edges terminating at the focal node-was the most appropriate centrality measure in such a network, and we follow their method here. An example turn-based network can be found in Figure 2.

\section{Interruption Networks}

What we propose as an "interruption network" is the representation, for a given group, of all instances of simultaneous speech between members of the group during the observation window. If only ISS is considered, an edge exists from node $i$, representing person $\mathrm{A}$, to node $j$, representing person $\mathrm{B}$, if, during the observation window, $\mathrm{B}$ interrupted A (Figure 1). An ISS-only network therefore corresponds to the colloquial understanding of interruptions, and the weight on the edge $i \rightarrow j$ is equal to the count of instances in which B interrupted A. If non-interruptive simultaneous speech (NSS) is considered, an instance of NSS adds an edge, or weight to an existing edge, going from person $C$ to person D if person C engaged in NSS within D's speaking turn. Note that ISS edges and NSS edges point in opposite directions from the perspective of the person engaging in the behavior (either ISS or NSS), but both point towards the person who "held the floor" at the conclusion of the behavior (Figure 1). Either ISS, NSS, or both may be used to construct an interruption network, and the networks corresponding to each construction method for an example group can be found in the top row of Figure 2.

As with turn-based networks, network metrics in interruption networks have direct behavioral interpretations. For example, the non-normalized in-degree centrality of each node in an ISS-only interruption network is the count of interruptions made by the represented individual; non-normalized out-degree centrality is the number of times that individual was himself/herself interrupted. Individuals who either do not engage in the conversation, or do so without interrupting or being interrupted, are represented by isolated nodes. The network also has a local ordering, such that successor nodes "held the floor" in an interraction with predecessor nodes, suggesting that a node that has predecessors but no successors "held the floor" the most, or was able to do so with another individual who otherwise did so. Eigenvector centrality measures capture this idea well, and we use PageRank centrality to account for isolated nodes (Langville \& Meyer, 2005). We propose this measure of importance here as theoretically derived and develop empirical evidence to support its use in this study.

Note that interruption networks as proposed here, as well as turn-based networks, are static networks derived from temporal data, in both cases by taking the count of events in a certain time window as a weight on a static edge (Holme \& Saramäki, 2012). Analysis of 
contact sequences or other temporal network representations are possible; however, prior research in this area has typically considered cumulative durations or counts of discrete behaviors, so for the sake of comparison that is the approach taken here.

\section{Hypotheses}

The evidence for the association between interruptions and status is sometimes presented as a given (Smith-Lovin \& Brody, 1989) — and indeed, given the boundary conditions discussed above, there is no lack of evidence to support this claim (Hall et al., 2005). To highlight an experimental example, Farley (2008) had adult volunteers interact with a confederate who, according to a randomized condition, either waited for the participant to finish each statement or interrupted many of the participants statements. Participants in the interrupted condition rated themselves lower on influence and the confederate higher on dominance, and observers appeared to rate the participant and confederate according to similar patterns. When it comes to informal or ad hoc groups, and given the literature review and theoretical development above, it seems reasonably secure that individuals who interrupt more are subsequently more likely to be attributed social status.

Hypothesis 1a: At the individual level, the total number of interruptions positively predicts assessed social status in informal groups.

However, as noted earlier the field-based literature presents mixed results with regard to the relationship between interruptions on social status, and laboratory-based literature provides limited information about the effect of statistically controlling for speaking time. For example, Hall and Friedman (1999) present a study emblematic of the influence of the study location moderator in the Hall et al. (2005) meta-analysis: Hall and Friedman (1999) found that in ad hoc groups of organizational incumbents high status individuals speak less and interrupt less when status differences are greater; the reverse trend was found for their lower status conversation partners. Unfortunately, speaking time and interruption counts were not directly compared at the individual level, nor was the association between interruptions and status calculated while controlling for speaking time. Intead, interruptions and speaking time appear to be "moving" together in the Hall and Friedman (1999) study, suggesting common correlation. Observational studies in the laboratory have found similar patterns, including finding a relationship between a count of interruptions and other, apparently contradictory formulations such as "yielding to interruptions" and "overcoming interruptions" (Ng, Brooke, \& Dunne, 1995).

A few studies have controlled for speaking time in regression or partial correlation analysis. Dabbs, Ruback, and colleagues investigated group speech patterns in a series of observational studies in the 1980s (Dabbs \& Ruback, 1984, 1987; Dabbs et al., 1987; Ruback, Dabbs, \& Hopper, 1984): these studies showed a tendency for near-zero, non-significant correlations between both types of simultaneous speech and participant ratings of leadership and related scales when controlling for speaking time with partial correlations. However, Feldman and Gill (2019), analyzing United States Supreme Court transcripts, did find an association between status and interruptions when controlling for speaking time, in this case represented by its transcript-based proxy, word count. In the Feldman and Gill (2019) study, interruptions were more likely when the interrupting justice 
had higher tenure than the interrupted justice, when controlling for the number of words spoken in the focal dyad.

Thus, it is not immediately clear to what extent the mechanistic linkage between speaking time and interruptions (one must speak to interrupt) is statistically disguising or revealing important relationships between behaviors and attributions. Given the weight of evidence, it seems conservative to expect that the inclusion of speaking time as a control will at least attenuate the effect of counted interruptions on predictions of subsequent status attributions.

Hypothesis 1b: At the individual level, when controlling for speaking time the total number of interruptions does not positively predict sociometric status in informal groups.

As discussed above, our intuition is that accounting for the directed aspect of simultaneous speech by means of an interruption network may better reflect the status dimension of speaking behaviors if such information is in fact encoded in simultaneous speech. In order to support the internal consistency/validity of such an analytical strategy, several alternative hypotheses need to be considered.

First, it is possible that interruptions are a random event based on two individuals speaking in close proximity in time. This is not an unreasonable idea: a large survey of conversations from several interaction domains and languages found an approximately normal distribution of inter-event times at speech boundaries, with the mean inter-event time being slightly positive (Heldner \& Edlund, 2010). Stated differently, the expectation is that there will be a slight pause between speakers, but longer pauses and "negative" pauses (i.e., the second speaker beginning her turn while the first is still speaking) are both common. Taking this random event hypothesis as the null, then, we hypothesize the alternative: that the "direction" of a simultaneous speech event-who interrupts whom, or who "fails" to interrupt whom-carries meaning over and above an association of speakers in time.

Hypothesis 2: The "direction" of a simultaneous speech event is not predictable from speaking time alone.

Second, status is thought to be an ordered construct: some individuals "endorse" another as having higher status, individuals vary in the quantity and strength of these "endorsements," and endorsements carry more weight if they come from those with many or strong endorsements themselves (Kawakatsu, Chodrow, Eikmeier, \& Larremore, 2021). Such a phenomenon should lead to a hierarchical network such that the so-called transitive or "feed forward" triads are relatively more common than intransitive or "feedback" triads (Milo et al., 2002). Indeed, such mesoscale structures have been found in hierarchical social networks in a variety of situations (Kawakatsu et al., 2021). If interruption networks are reflecting status in some way, interruption networks should also display mesoscale structures, or motifs, of these types.

Hypothesis 3: Interruption networks have a hierarchical internal ordering.

Third, if simultaneous speech contains information distinct from that found in speaking time, then networks built from simultaneous speech should differ from networks built from 
other aspects of speech-even though the building blocks of both types of networks correlate with speaking time. Networks based on speaking turns (Sauer \& Kauffeld, 2013) provide such a comparator in that both speaking turns and simultaneous speech are highly correlated with overall speaking time, but use non-overlapping information from the speech activity data of each individual. If simultaneous speech is different from speaking time generally, then different aspects of the information environment should predict edge formation in networks based on these disparate variables.

Hypothesis 4: Predictors of edge formation in interruption-based networks differ from predictors of edge formation in speaking turn-based networks.

If interruption networks have direction and a local ordering, then measures of importance on such networks should accommodate that flow: specifically, eigenvector centrality, or PageRank to accommodate isolated nodes. We can thus recast the question of whether or not interruptions, as discrete events, are important in predicting social status: does node importance in the network of directed simultaneous speech events predict social status? Using a network of discrete behaviors such as this has been used in ethological studies (Barrett, Henzi, \& Lusseau, 2012; DeDeo \& Hobson, 2021), but to our knowledge has not been used in management research.

A network analysis of simultaneous speech may predict assessed social status where simple counts of interruptions fail because counts of interruptions focus solely on the sender of the interruption (or simultaneous speech behavior generally), ignoring who was or was not interrupted. For example, if one particular individual interrupts all others save one, and that one can interrupt the main interruptor, a simple count of interruptions finds the main interruptor the most important—an interruption network approach considers that the one who interrupts the main interruptor is more important. If this latter interpretation is correct, as other research on hierarchies in social structure suggests (DeDeo \& Hobson, 2021), then the interruption network will capture more of the available information about who is related to whom how in a status dimension than a simple count of interruptions will. Our central hypothesis, then, is that importance in interruption networks predicts attributions of social status even when controlling for speaking time.

Hypothesis 5: When controlling for speaking time, centrality in interruption networks positively predicts sociometric status in informal groups.

\section{Methods}

\section{Data}

The data for this study is publicly available (https://orb.binghamton.edu/management_fac/2/) and was first reported in MacLaren et al. (2020). We use this data for the present study for several reasons. First, the data is public: other researchers may check our analyses and try their own. Second, summary statistics and model results have been previously published, meaning that the results of the current study can be compared against the published record. Third, the data has information, such as both diarized speaking data and leadership attribution scores, not to mention other psychometric covariates, not commonly found in combination in other corpora. 
The diarization data comes from 10-minute video segments recording discussions from 33 ad hoc student groups ( $\bar{n}=7.79$, range $4-10)$ involved in planning for a low-fidelity computer-mediated task. There were two tasks, one military and one business, and two locations (S1, with 100 participants in 11 groups, and S2, with 156 participants in 22 groups); differences in the relationship between speaking time and leader emergence across these contrasts were not significant in MacLaren et al. (2020). An "operator" was randomly assigned to implement group decisions in the computer program itself, but participants were instructed not to consider the operator as having any special leadership role.

Participants completed psychometric instruments, practiced the task as an individual and as a group, and were given 10 minutes to plan their approach to the task; after voting on emergent leaders during the planning phase, the groups had 60 minutes to complete the task. Several psychometric and demographic instruments were also administered; participants were older and more likely to speak English as a second language at S1, but otherwise did not differ greatly between locations MacLaren et al. (2020).

The data analyzed here comes from the 10-minute planning phase. Audiovisual data was collected by means of two Canon VIXIA HF-series cameras not assisted by external microphones. Diarization was done by hand using the ELAN video annotation software (Max Planck Institute for Psycholinguistics, 2018). To gather data for this study from the existing diarizations, Python scripts were written that operationalized simultaneous speech according to the definitions given above, tabulated both kinds of simultaneous speech from the diarization data files, and transformed that data into interruption networks. Turn-based networks and vote networks were constructed in a similar way. The Python scripts that accomplish these tasks are available, along with the rest of the data processing and analysis script files in Python, R, and Stata associated with this study, at https://github.com/ngmaclaren/interruption-networks.

\section{Social Status}

The criterion variable of interest in this study is social status. Social status in informal groups has been assessed in a variety of ways, including a variety of self- and other-report survey items. Hall et al. (2005) argued that the constructs these survey items represent lack discriminant validity and should be considered part of a "verticality" dimension, itself defined similarly to sociometric status (cf, Anderson et al., 2015; Bendersky \& Pai, 2018; Hall et al., 2005). Whatever the case may be, it is clear that self-report items with face validity to a variety of theoretical constructs have similar empirical correlations with speaking time, itself used most commonly to predict leader emergence (MacLaren et al., 2020; Schmid Mast, 2002).

Face valid leader nominations have also been used in at least two ways: first, to identify single leaders in a given group, usually by identifying the individual who received the most nominations; and second, to establish "leadership networks," thought to represent patterns of leadership in informal groups (Carter et al., 2015). Ratings of leadership and related traits, perceived behaviors, and other qualities have also been used as indicators of both leader emergence and sociometric status (Hall et al., 2005; MacLaren et al., 2020). Given this variety of operationalizations and lack of clear construct differentiation, we used the leader nominations in the MacLaren et al. (2020) data as an indicator of social status, recognizing that this operationalization of social status may suffer from construct deficiency. 
Leadership attributions came in the form of nominations in a single item issued to the participants immediately following the recorded segments: "We would like you to nominate an individual or multiple individuals that emerged as a leader or leaders during this planning (gameplay) phase. You can select as many as five leaders or as few as one. You will be choosing the leader or leaders based on workstations. Please take a moment to nominate the leader or leaders of the group." Participants entered work station numbers without additional weights or other modifications, and all votes were treated equally in this analysis.

\section{Two-Stage Least Squares Regression}

Hypotheses 1 and 5 are about predicting an individual attribute (social status, operationalized as leader emergence votes) from a variety of exogenous and endogenous predictors. As MacLaren et al. (2020) argued, speaking time can be considered endogenous to the group setting, motivating the use of two-stage least squares (2SLS; Antonakis, Bendahan, Jacquart, \& Lalive, 2010; Wooldridge, 2010). MacLaren et al. (2020) also presented evidence that the individual level of analysis was appropriate to the question and data considered, though cluster-robust standard errors were used as a conservative approach to account for group membership. The Lewbel method (Lewbel, 2012) was used to improve estimation given weak instruments and substantial heteroscedasticity.

Simultaneous speech, by the same logic, is also endogenous to the group setting: there may be some features that predict simultaneous speech, but simultaneous speech observed at a given time within a given group is dependent on influences unique to that setting, not just to exogenous predictors such as gender or age. In the present study, we begin with the MacLaren et al. (2020) model and data, replacing speaking time with the count of interruptive simultaneous speech associated with each group member. This approach treats interruptions as an "undirected" quantity: that is, only the number of interruptive simultaneous speech (ISS) events initiated by the focal group member were counted, no matter to whom the event was directed. The two equations of this modified model were:

$$
\begin{gathered}
I S S=\beta_{0}+\beta_{i} X_{i}+\beta_{i} Z_{i}+e \\
\text { LeaderEmergence }=\gamma_{0}+\gamma_{1} \widehat{I S S}+\gamma_{i} X_{i}+u
\end{gathered}
$$

where the $X_{i}$ are the exogenous variables (age, gender, game knowledge, self-reported status as a native English speaker, group size, simulation, and institution), the $Z_{i}$ are the instrumental variables (intelligence, status as the randomly assigned game operator, and five factor personality scores), and $e$ and $u$ are errors (MacLaren et al., 2020). If $\gamma_{1}$ in eq. 2 is positive and significant, it suggests that, controlling for a variety of individual and contextual variables and the endogeneity of interruptions, the count of interruptions a group member makes during an observation period predicts the count of leader emergence votes that participant receives immediately after the observation period is ended.

A similar approach is used to control for speaking time, except there are two Stage 1 equations - the equation predicting ISS given above and the equivalent equation predicting 
total speaking time (TST) in MacLaren et al. (2020)—and two endogenous variables in Stage 2:

$$
\begin{gathered}
I S S=\alpha_{0}+\alpha_{i} X_{i}+\alpha_{i} Z_{i}+e_{I S S} \\
T S T=\beta_{0}+\beta_{i} X_{i}+\beta_{i} Z_{i}+e_{T S T} \\
\text { LeaderEmergence }=\gamma_{0}+\gamma_{1} \widehat{I S S}+\gamma_{2} \widehat{T S T}+\gamma_{i} X_{i}+u
\end{gathered}
$$

If $\gamma_{1}$ in eq. 5 is positive and significant given that speaking time is also in the model, then that would indicate support for the idea that simultaneous speech is associated with leader emergence over and above the effect of speaking time.

Hypothesis 5 requires models in which network centrality (PageRank) is included as an endogeneous predictor. This is a hypothesis about nodes, relying on edge information, as opposed to a hypothesis about edges, for which exponential random graph model (ERGM) or stochastic actor-oriented model (SAOM) approaches would be more appropriate (Snijders, Van de Bunt, \& Steglich, 2010). Each individual's PageRank is by definition dependent on global information in the network, and observations are not independent in that, for example, changing the in-degree of node $i$ affects not only $i$ 's PageRank but also that of node $j$. Autologistic actor attribute models are argued to address this concern (Parker, Pallotti, \& Lomi, 2021) but to our knowledge have not been extended to the types of networks (i.e., weighted edges) or outcomes (i.e., count variables as opposed to binary) considered here. However, in a regression framework the primary concern is independence of errors given the model, and here the 2SLS framework appears useful: as with speaking time, an individual's PageRank will be estimated based on available information, rather than using PageRank values directly.

\section{Simulation Studies}

\section{Interruption Direction}

Hypothesis 2 suggests that if interruptions are an important directed behavior and not, as a null hypothesis might suggest, a random event based on co-occurrence of speaking between two participants, then changing the direction of the edges in a given network should change the calculations made from the network in a meaningful way. Because eigenvector centrality measures take the entire network into account when assigning a centrality score, substantive changes in the network brought about by randomly changing edge directions should be captured in changes in the node with the maximum PageRank value.

The algorithm to randomize edge directions is as follows:

1. Generate a list of node pairs and all edges (including associated weights) between 
each node pair.

2. For each node pair, with probability $p$ reverse the source and target of each edge between the two nodes (maximum of two), leaving the weight in place, such that $(i, j, w)$ becomes $(j, i, w)$. Otherwise, do nothing.

3. Continue the algorithm until each pair of connected nodes in the original graph is considered.

A set of 100 random networks was generated according to the above algorithm for each empirical network. The "leader" of each randomized network (that is, the node with the maximum PageRank centrality) was compared against the leader from the empirical network, and the proportion of matches between the randomized and empirical networks recorded. The probability of reversing an edge was changed for each set of simulations, from $p=0.01$ to $p=1$.

\section{Network Motifs}

Hypothesis 3 posits the idea that mesoscale structures, also called motifs, of interruption networks will suggest an internal structure consistent with a status-based interpretation of the information from which the network is drawn. A feature of network analysis is the ability to represent widely different systems with a common mathematical framework (Sayama et al., 2016). Milo et al. (2002) provided evidence that this translation was not only terminological, but apparently structural as well: information processing networks, such as gene transcription networks and networks of electronic circuits, shared common substructures lacking from networks representing an inherent hierarchy, such as food webs - and vice versa. Thus, widely different systems may be compared functionally by examining the small substructures that comprise them. Wasserman and Faust (1994) held a similar belief, focusing on what they called the transitive triads - those in which A $\rightarrow \mathrm{B} \rightarrow \mathrm{C}$ and $\mathrm{A} \rightarrow \mathrm{C}$-as an important triad in social networks, capturing the idea that a friend of a friend is a friend.

Statistical analysis of triad prevalence requires comparing the observed counts with an appropriate distribution of counts from randomized networks. Milo et al. (2002) and others (e.g., Holme \& Saramäki, 2012) advocate using an edge randomization procedure, simulated many times, to build an empirical null distribution, similar in sense to a bootstrapping procedure, then comparing the observed, empirical values to the "bootstrapped" values in a permutation test. We used the (Holme \& Saramäki, 2012) algorithm to randomize edges as follows:

1. Select two edges, $(i, j)$ and $(k, l)$.

2. Swap the endpoints of the edges randomly according to either $2 \mathrm{a}$ or $2 \mathrm{~b}$, below, with equal probability:

(a) $(i, l)$ and $(k, j)$, or

(b) $(i, k)$ and $(l, j)$. 
3. If the previous step created a self- or a multiple edge, undo the change and select another edge $\left(k^{\prime}, l^{\prime}\right)$.

4. Continue the algorithm until every pair of edges in the original contact sequence has been considered.

Randomizing the edges in this way preserves the order of events in the observation window, represented by edges, while shuffling the nodes the edges connect.

As before, 100 randomized networks were generated for each empirical network. Given the small size of the graphs, only triads (that is, not tetrads or higher order substructures) were counted. In each empirical and randomized graphs, the triad census was taken (Wasserman \& Faust, 1994). A directed permutation test was applied to each, with the $\mathrm{p}$-value calculated as the proportion of triads in the randomized networks that appeared more times in the randomized data than in the respective empirical network. Following Milo et al. (2002), we apply a correction for multiple comparisons when assessing $p$-values in this analysis: we use a Bonferroni correction for a target of $\alpha=0.05$ and 16 null hypothesis tests, suggesting an effective $\alpha$ of $\frac{\alpha}{16}=0.003$.

\section{Exponential Random Graph Models}

Hypothesis 4 concerns different predictors of edge formation in alternative network representations of speech activity - this hypothesis does involve an edge-based outcome variable, and we use ERGMs to test it. The intent of exponential random graph modeling is to explicitly model the endogeneity associated with networks of relationships (Goodreau, Kitts, \& Morris, 2009); for example, by considering the effects of the presence of a given number of mutual ties on the probability of observing another tie. Predictor variable values used in these models are set either before the modeled interactions (e.g., gender) are are thus exogenous, or simultaneously with the modeled interactions (e.g., speaking time) within the limits of this analysis.

Networks were built separately for interruption networks (using both ISS and NSS to form edge weights according to the rules described above) and turn-based networks. To address these potential small-sample biases within groups, data from all groups were included in one ERGM model for each network type, using structural zeros to forbid estimation of edges between groups. Weighted (or valued) edges were used in both cases (Krivitsky, 2012); we used a discrete reference distribution that is within statistical error of the observed data to approximate the heavy-tailed expected distribution of edge weights in these networks. The network statistics for the ERGMs used in this study are in Table 1. Note that many network statistics used in unweighted networks, such as several of those associated with triad analysis, are not implemented for valued networks in the relevant software.

\section{Results}

Summary statistics for the main variables considered in this study are in Table 2. Simultaneous speech is present in all groups (ISS: $\min =7, \max =156$; NSS: $\min =3$, $\max$ 
Table 1: Exponential Random Graph Network Statistics

\begin{tabular}{ll}
\hline Category & Description \\
\hline Density & Either a count of edges or a sum of edge weights. \\
Mutuality & The count of edges or sum of edge weights for dyads for which both \\
& $i \rightarrow j$ and $j \rightarrow i$ exist, using the smaller of the two edge weights to \\
& represent the pair in a weighted network (see Krivitsky, 2012). \\
Transitive Triads & A count of one of the so-called transitive triads, in which a path \\
& $i \rightarrow j \rightarrow k$ and $i \rightarrow k$ exists (see Wasserman \& Faust, 1994). A \\
& weighted version of this statistic is also available (see Krivitsky, \\
Node Covariates & $\begin{array}{l}\text { Edges are counted or summed if an attribute of either the source or } \\
\text { target node meets a given criteria. Criteria can be numeric or } \\
\text { categorical. Although the number of in-edges equals the number of } \\
\text { out-edges in a given network, nodal attributes may be associated with } \\
\text { different in-edge and out-edge degree histograms. } \\
\text { Edges are counted or summed if both the source and target node are } \\
\text { equivalent on a given attribute. }\end{array}$ \\
\hline
\end{tabular}

Note: Categories of network statistics used in this study, along with brief descriptions. For further discussion, see Morris, Handcock, and Hunter (2008) and Krivitsky (2012).

$=71$ ) but ICC1 values support the idea that simultaneous speech is more variable between groups than within (ISS $=0.30, \mathrm{NSS}=0.23$ ). Speaking turns and TST are highly variable within groups but similar between groups (Table 2). At the individual level, all speaking behaviors considered in this study are highly correlated, and each is at least moderately correlated with leader emergence votes. Speaking turns $(r=0.17)$, TST $(r=0.24)$, and leader emergence votes $(r=0.34)$ are moderately correlated with male gender, but ISS ( $r=$ $0.07)$ and NSS $(r=0.00)$ are not. Thus, despite high correlations between simultaneous speech behaviors and speaking time, simultaneous speech appears to be distributed in a different manner between groups than speaking time is and has different relationships with other variables, such as gender.

Although some studies have in the past advocated for expressing the number of instances of a behavior during group work as a rate against speaking time (e.g., Bavelas et al., 1965), doing so with simultaneous speech may lead to inappropriate conclusions. In Figure 3 it can be seen that both the highest and lowest individual level interruption rates are to be found in the participants who speak the least and are the least likely to be identified by group members as emergent leaders. While it may be the case that low interruption rates are associated with leader emergence, this appears to be mostly due to the relative infrequency of interruptive speaking events with increased speaking time. Analyzing speaking time and simultaneous speech as distinct variables - as opposed to considering a rate of simultaneous speech or ignoring it altogether-thus appears to be supported in this data. 


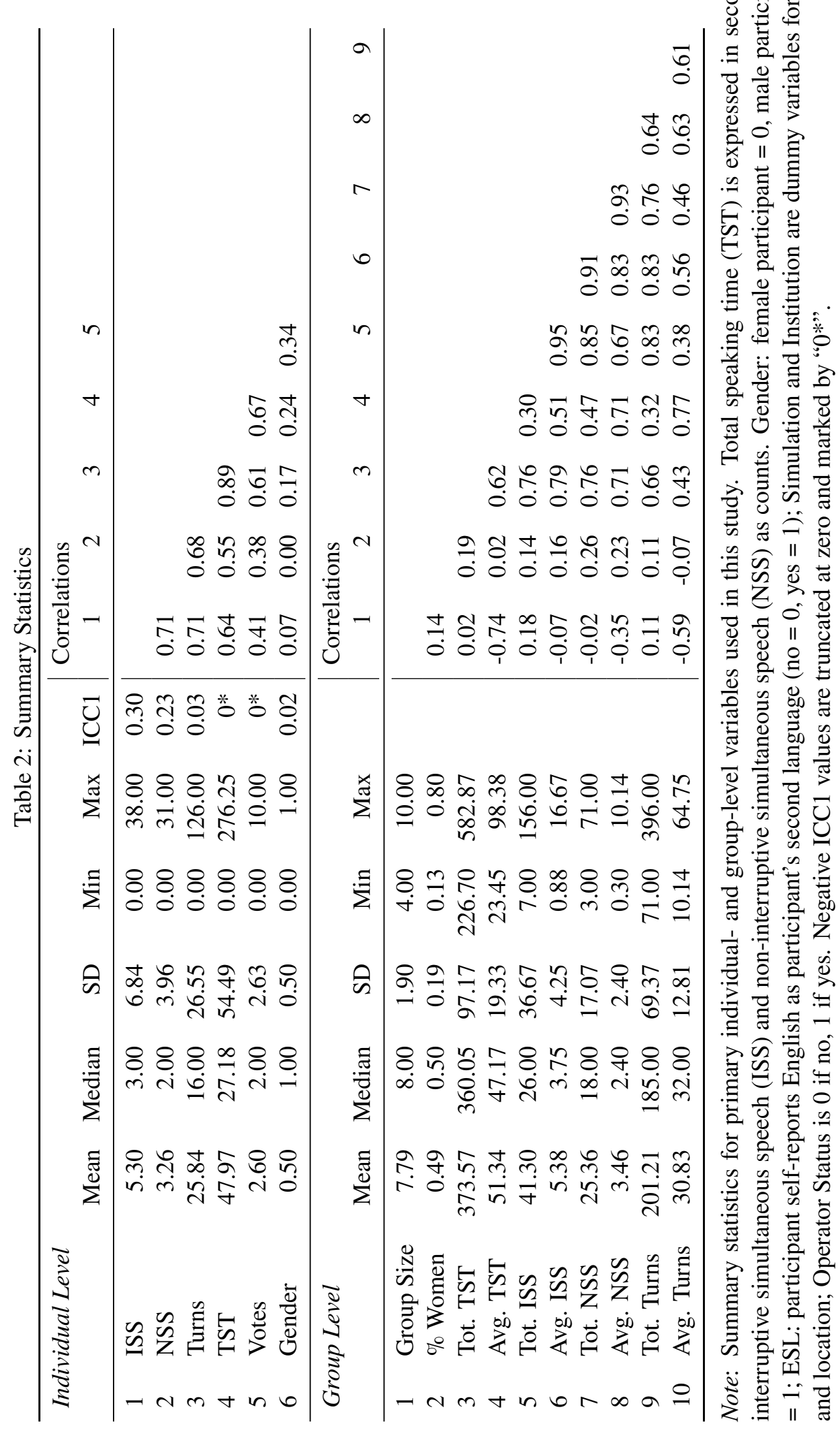




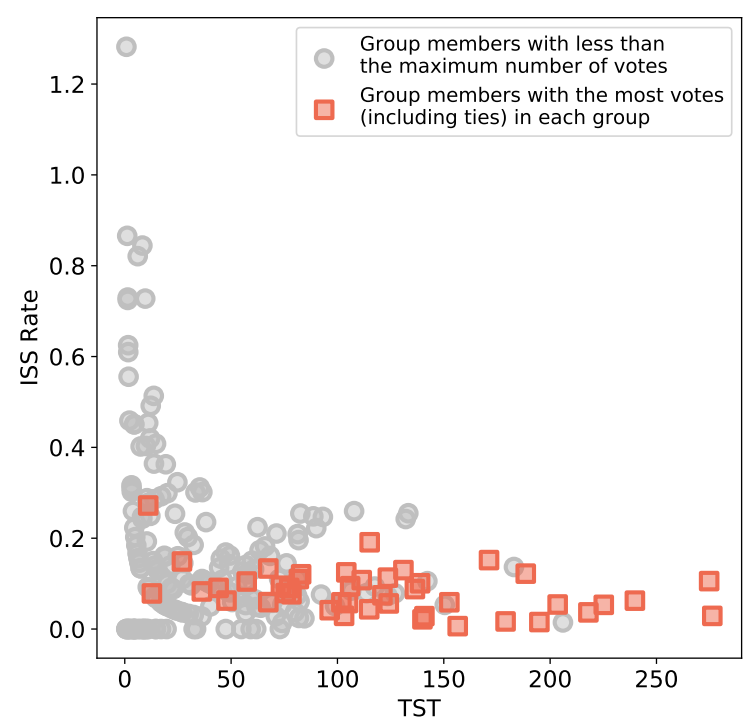

Note: Interruptive simultaneous speech (ISS) expressed as a rate relative to total speaking time (TST). The ISS rate is the number of interruptions per second of speaking time. Square points in contrasting color represent participants with the highest number of votes in each group, including ties.

\section{Interruptions as a Predictor of Leader Emergence}

As others have found (Hall et al., 2005), ISS predicts an attributional assessment of within-group social status (Table $3 ; b=0.106, p=0.000$ ), supporting Hypothesis 1a. Also consistent with prior research (Dabbs \& Ruback, 1987; Dabbs et al., 1987), including speaking time as a control attenuates the observable effect of ISS on assessed social status ( $b=-0.031, p=0.220$ ) even though total $R^{2}$ increases and other model selection criteria are largely similar (Table 3 ). Thus, Hypothesis $1 \mathrm{~b}$ is also supported. These findings support the statistical importance of speaking time in predicting within-group social status and do not strongly support the inclusion of counts of ISS in such models. However, the purpose here is not to better predict assessments of social status, but instead to develop a model of within-group relationships based on speaking behaviors. To that end, it is important to note that the evidence that ISS negatively predicts social status, perhaps because of interruptive behaviors are negatively valenced, is not strong in this data.

\section{Internal Consistency of Interruption Networks}

\section{Randomizing Edge Direction}

Introducing randomness into the direction of interruption edges decreases the average number of matches between the randomized network "leaders" (those with the highest PageRank) and the empirical "leaders" (Figure 4). In the ISS-only networks, the mean proportion of matches at $\operatorname{Pr}($ Reverse Edge $)=0.5$ is about 0.67 , suggesting that about one 
Table 3: Predicting Leader Emergence from ISS

\begin{tabular}{|c|c|c|c|c|c|c|}
\hline Leader Emergence & $b$ & SE & $p$ & $b$ & SE & $p$ \\
\hline$\widehat{I S S}$ & 0.106 & 0.028 & 0.000 & -0.031 & 0.025 & 0.220 \\
\hline$\widehat{T S T}$ & & & & 0.029 & 0.005 & 0.000 \\
\hline Gender & 1.694 & 0.227 & 0.000 & 1.092 & 0.191 & 0.000 \\
\hline Age & 0.013 & 0.055 & 0.814 & -0.035 & 0.046 & 0.438 \\
\hline Game Knowledge & 0.149 & 0.058 & 0.011 & 0.081 & 0.043 & 0.058 \\
\hline ESL & -0.562 & 0.379 & 0.138 & -0.520 & 0.310 & 0.094 \\
\hline Group Size & 0.345 & 0.084 & 0.000 & 0.483 & 0.060 & 0.000 \\
\hline Simulation & 0.259 & 0.223 & 0.246 & 0.228 & 0.182 & 0.211 \\
\hline Institution & 0.510 & 0.434 & 0.240 & 0.132 & 0.298 & 0.659 \\
\hline Constant & -3.263 & 1.752 & 0.063 & -3.042 & 1.134 & 0.007 \\
\hline & \multicolumn{3}{|c|}{$\begin{array}{l}\text { DWH } F(1,32)=5.336, p=0.028 \\
\text { Hansen's } J=15.517, p=0.276\end{array}$} & \multicolumn{3}{|c|}{$\begin{array}{l}\text { DWH } F(2,32)=3.777, p=0.034 \\
\text { Hansen's } J=16.535, p=0.621\end{array}$} \\
\hline & \multicolumn{3}{|c|}{$\begin{array}{l}\text { Test of Excluded Instruments } \\
F(14,32)=20.79\end{array}$} & \multicolumn{3}{|c|}{$\begin{array}{l}\text { Test of Excluded Instruments } \\
F(21,32): \text { ISS }=48.53, \text { TST }=57.97\end{array}$} \\
\hline
\end{tabular}

Note: Regression results for the two-stage least squares models predicting leader emergence with interruptive simultaneous speech with (right) and without (left) speaking time. Lewbel estimation was used in both models; first stage estimates are not shown. TST: total speaking time; ISS: interruptive simultaneous speech. Gender: female participant $=0$, male participant = 1; ESL: participant self-reports English as participant's second language (no $=0$, yes = 1); Simulation and Institution are dummy variables for task and location as described in MacLaren et al. (2020); Operator Status is 0 if no, 1 if yes; DWH: Durbin-Wu-Hausman. 
Figure 4: Randomized Edge Directions
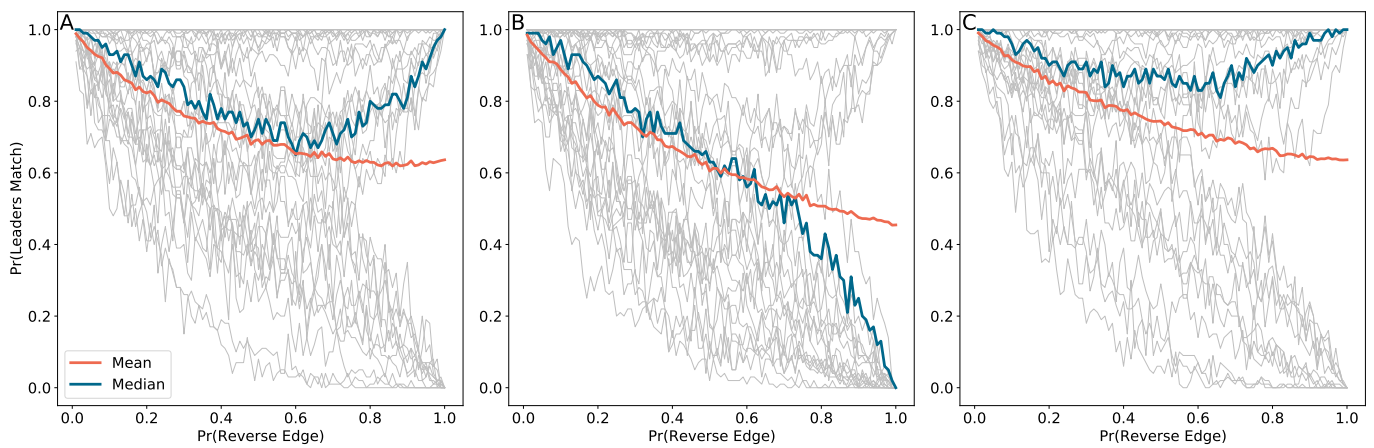

Note: Simulation results from randomly switching the direction of a selected edge in interruption networks containing (A) only interruptive simultaneous speech (ISS), (B) only non-interruptive simultaneous speech (NSS), and (C) networks containing both ISS and noninterruptive simultaneous speech. Edges were reversed with probability $p$ in 0.01 increments between 0.01 and 1.00 (x-axis). Each group was simulated separately, shown by the gray lines. The y-axis represents the proportion, of 100 simulated trials, in which the node with the maximum PageRank in the randomized network matched the node with the maximum PageRank in the empirical network.

third of the variance in leader identification by the PageRank method is due to the direction of the within-dyad edges. However, there is substantial variability between networks, with about one third of networks having an approximately monotonic decrease in the proportion of matches between randomized and empirical leaders; about one third showing a U-shaped relationship between switching probability and matching; and about one third being either little influenced by randomizing edge directions or not at all. Trends in the mean proportion of matches have a similar shape in all three ways of constructing interruption networks; the networks containing both ISS and NSS are the least altered by the simulation but still show a reduced average match between simulated and empirical networks with an increasing probability of switching the direction of an edge. At the sample level the direction of the edge clearly matters, although node activity (speaking time) retains its importance.

\section{Network Motifs}

The data summarizing the network motifs analysis can be found in Figure 5. Using the conservative Bonferroni approach the important triads in the networks are $021 \mathrm{C}$ for ISS-only networks, 030T for NSS-only, and 120D in networks that contain both types of simultaneous speech. If the Bonferroni correction is considered too conservative, the Holm-Bonferroni method would add triad 030T to the list of significant triads for the ISS-only networks.

First, it is important to note there is little support for the importance of cyclic triads, supporting the idea that these networks have an essentially hierarchical structure at the mesoscopic level. Second, transitivity appears to be important in interruption networks, although the interpretation may be not so much "a friend of a friend is a friend" but rather an indication that individuals appear to be interacting with each other in consistent ways. Assume again that nodes $i, j$, and $k$ represent individuals $\mathrm{A}, \mathrm{B}$, and $\mathrm{C}$, and that $030 \mathrm{~T}$ 
Figure 5: Network Motifs

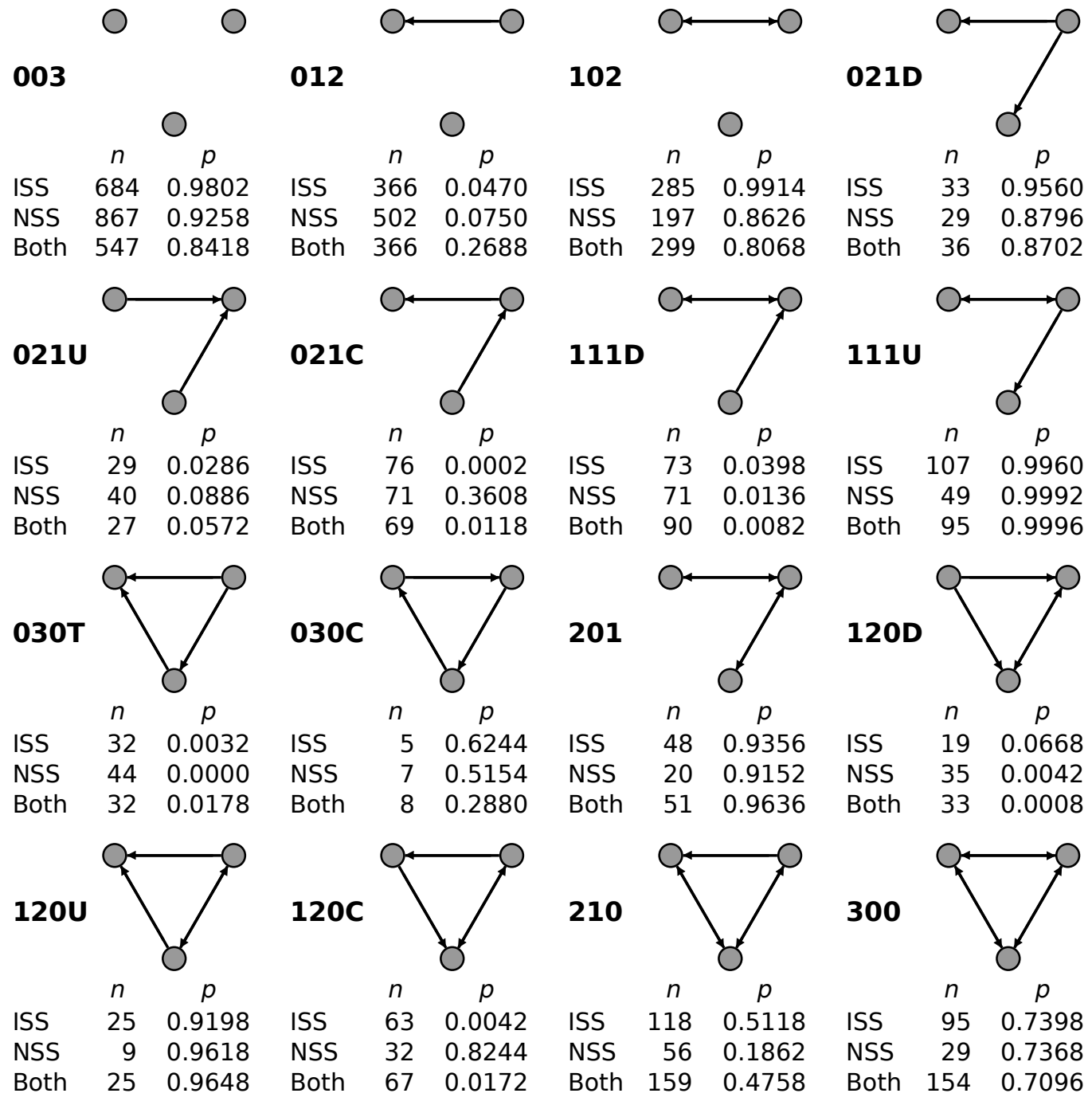

Note: Each triad from the standard triad census (Wasserman \& Faust, 1994) is shown along with the number, $n$, of times that triad appeared in the empirical graphs and the proportion, $p$, of simulation runs in which the simulated triad count was more than the empirical count. For example, triad $021 \mathrm{C}$ appeared more often in the empirical ISS networks than in all but 14 of the 5000 simulation runs, whereas triad 111D appeared more often in the simulated ISS networks 4014 times. 
indicates that $i \rightarrow k \mid i \rightarrow j \rightarrow k \in G$, with $G$ being the NSS-only interruption network. That this structure is found significantly more often than chance suggests that $\mathrm{C}$ and $\mathrm{B}$ "resisted" interruptions from A, and C "resisted" an interruption from B, but that neither A nor B were observed to "resist" an interruption from C. A pattern such as this is consistent with the interpretation of simultaneous speech as indicating relative status. A similar interpretation holds for the "line" motif, triad 021C, but is more clearly hierarchical (Milo et al., 2002). 120D can be interpreted as following a similar pattern, but with more edges due to the interruption networks with both types of edges have a higher density (Figure 5).

\section{Divergence between Network Representations of Conversations}

Equivalent exponential random graph models were fit in the interruption networks using both ISS and NSS and in the turn-based networks: if predictors of tie formation differ between these network types, it should be apparent in such models (Table 4).

The ERGM analysis reveals several important similarities between interruption networks and turn-based networks. For example, there is a strong, positive trend towards transitivity: triads including the motif $i \rightarrow k \mid i \rightarrow j \rightarrow k$ are more likely than predicted by chance in both networks. Additionally, speaking time emerges as an important control on both in-edges and out-edges in both ERGMs. In general, five-factor personality model variables do not have important effects in these models, although there are exceptions (e.g., openness to experience negatively predicts out-edge weight in the interruption networks; $b$ $=-0.159, p=0.001$ ). Status as a non-native English speaker is negatively associated with edge weight in both networks.

However, differences between the two network types in this analysis are present and point to different predictors of the different behaviors (Table 4). For example, there appears to be a strong positive trend for mutuality in the turn-based networks $(b=0.978, p=$ 0.000), indicating that if person A's turn follows person B's turn in a conversation, it is very likely that person B will at a different time also speak after person A. In interruption networks, there is a weak trend in the opposite direction such that if an interruption edge (indicating either ISS $i \rightarrow j$ or NSS $j \rightarrow i$ ) from A to B is less likely to be associated with an interruption edge $\mathrm{B}$ to A. Additionally, in the interruption networks, operator status is not strongly associated with edge weights, indicating that the simultaneous speech behaviors did not reliably differ between operators and other participants given their level of speaking and other controls. In the turn-based networks, however, there is a strong, positive association between operator status and both in- and out-edges: operators were more likely to speak frequently throughout the interaction period, leading to a greater probability of increased edge weight in turn-based networks.

Patterns relating to participant gender also differ between the network types. In turn-based networks, conversation is more likely to pass back and forth between speakers who share a gender ( $b=0.084, p=0.002)$; however, neither gender is more likely to "send" or "receive" edges (speaking turn transitions) in general. In interruption networks, on the other hand, men are less likely then women to "send" interruption edges (i.e., men are less likely to either be interrupted or to "fail" to interrupt others) but are neither more nor less likely to "receive" interruption edges (i.e., to interrupt others or to "resist" others' interruptions), nor are simultaneous speech behaviors more or less likely when both 
Table 4: Predictors of Edge Weight in Interruption and Turn-Based Networks

\begin{tabular}{|c|c|c|c|c|c|c|}
\hline & \multicolumn{3}{|c|}{ Interruption Networks (Both) } & \multicolumn{3}{|c|}{ Turn-Based Networks } \\
\hline & $b$ & SE & $p$ & $b$ & SE & $p$ \\
\hline \multicolumn{7}{|l|}{ Network Controls } \\
\hline Sum & -0.294 & 0.475 & 0.535 & -1.483 & 0.305 & 0.000 \\
\hline Mutuality & -0.175 & 0.101 & 0.083 & 0.978 & 0.075 & 0.000 \\
\hline Transitivity & 0.436 & 0.048 & 0.000 & 0.083 & 0.021 & 0.000 \\
\hline \multicolumn{7}{|l|}{ In-Edges } \\
\hline TST & 0.007 & 0.000 & 0.000 & 0.007 & 0.000 & 0.000 \\
\hline Intelligence & -0.002 & 0.004 & 0.639 & -0.003 & 0.003 & 0.333 \\
\hline Game Knowledge & -0.005 & 0.015 & 0.719 & 0.015 & 0.013 & 0.233 \\
\hline Conscientiousness & 0.017 & 0.049 & 0.729 & 0.060 & 0.044 & 0.177 \\
\hline Agreeableness & 0.019 & 0.045 & 0.678 & -0.026 & 0.043 & 0.541 \\
\hline Neuroticism & -0.028 & 0.038 & 0.461 & 0.004 & 0.035 & 0.919 \\
\hline Openness & -0.029 & 0.047 & 0.536 & -0.039 & 0.041 & 0.338 \\
\hline Extraversion & 0.001 & 0.043 & 0.973 & -0.006 & 0.043 & 0.886 \\
\hline Gender & -0.062 & 0.053 & 0.239 & -0.005 & 0.048 & 0.913 \\
\hline ESL & -0.253 & 0.074 & 0.001 & -0.243 & 0.063 & 0.000 \\
\hline Operator & -0.042 & 0.070 & 0.552 & 0.377 & 0.054 & 0.000 \\
\hline \multicolumn{7}{|l|}{ Out Edges } \\
\hline TST & 0.007 & 0.000 & 0.000 & 0.008 & 0.000 & 0.000 \\
\hline Intelligence & -0.012 & 0.004 & 0.002 & -0.004 & 0.004 & 0.258 \\
\hline Game Knowledge & -0.028 & 0.013 & 0.038 & 0.019 & 0.013 & 0.125 \\
\hline Conscientiousness & -0.002 & 0.050 & 0.966 & 0.059 & 0.044 & 0.182 \\
\hline Agreeableness & -0.012 & 0.045 & 0.790 & -0.037 & 0.043 & 0.387 \\
\hline Neuroticism & -0.063 & 0.038 & 0.098 & -0.010 & 0.035 & 0.764 \\
\hline Openness & -0.159 & 0.046 & 0.001 & -0.062 & 0.040 & 0.121 \\
\hline Extraversion & -0.094 & 0.045 & 0.037 & -0.052 & 0.043 & 0.223 \\
\hline Gender & -0.141 & 0.050 & 0.005 & -0.024 & 0.047 & 0.608 \\
\hline ESL & -0.145 & 0.073 & 0.048 & -0.230 & 0.063 & 0.000 \\
\hline Operator & 0.098 & 0.064 & 0.127 & 0.295 & 0.055 & 0.000 \\
\hline \multicolumn{7}{|l|}{ Node Matching } \\
\hline Gender & 0.029 & 0.049 & 0.554 & 0.084 & 0.027 & 0.002 \\
\hline ESL & 0.032 & 0.070 & 0.646 & -0.012 & 0.041 & 0.769 \\
\hline
\end{tabular}

Note: Exponential random graph models for interruption networks using both interruptive and non-interruptive simultaneous speech (left) and turn-based networks (right). TST is total speaking time, ESL is English is a Second Language. 
speakers are of the same gender. In all, there are clear differences between ERGMs fitted to interruption networks and to turn-based networks in the same data.

\section{Centrality in Interruption Networks Predicts Within-Group Social Status}

An important question in this study is whether or not interruption networks can represent social status relationships in small groups. If centrality in such networks predicts assessed social status, such a view would be bolstered (Table 5). If only ISS ( $b=0.818, p=$ $0.761)$ or NSS ( $b=2.676, p=0.533$ ) is used to form the network, there is little support for the use of centrality in interruption networks to predict leader emergence votes. However, if both types of simultaneous speech behavior are accounted for, there is more support $(b=$ $6.522, p=0.085)$.

Although $p=0.085$ does not meet a $\alpha=0.05$ cutoff, other sources of information in this model, and in the results presented above, support the idea that PageRank centrality in interruption networks is an important predictor of assessed social status distinct from speaking time (Wasserstein \& Lazar, 2016). First, model selection criteria indicate that this model is correctly specified (Hansen's $J=25.156, p=0.156$ ) with strong instruments for both endogenous variables $(F(21,32)=11.07$ and 31.77 for centrality and speaking time, respectively). Model $R^{2}=0.804$, higher than any other model presented here or in prior work in the same data $\left(R^{2}=0.561\right.$ for speaking time as a single endogenous variable; MacLaren et al., 2020, p. 8), suggesting increased variance explained. Second, the coefficient on speaking time ( $b=0.016, p=0.010)$ is reduced, both from its value in ISS-only $(b=0.025, p=0.000)$ and NSS-only $(b=0.021, p=0.008)$ interruption network centrality models and from its previous estimate in the same data $(b=0.026, p<0.001$; MacLaren et al., 2020, p. 8). We conclude that speaking time remains an important predictor of assessed social status in small groups, but that centrality in interruption networks predicts assessed social status in small groups when controlling for speaking time.

\section{Discussion}

The primary purpose of this study was to further investigate the relationship between speaking time and within-group social status: if group members are "babbling," what are they doing while they are "babbling" that indicates social status? The evidence presented above suggests that at least one thing group members are doing is speaking over each other, or "resisting" such behavior in others, and that this is a behavior that has independent meaning with respect to attributed social status in informal groups. Prior research shows that controlling for speaking time reduces the statistical effect of interruptions (Dabbs et al., 1987); this, combined with evidence for a reversal in the relationship between counts of interruptions and social status in laboratory as opposed to field studies (Hall et al., 2005), questions the usefulness of simultaneous speech as an indicator of within-group social status. Our findings suggest that conflicts in prior literature may be due to ignoring the directedness of simultaneous speech: the data suggests that what matters for attributed social status is not necessarily how many times someone interrupted or "failed" to interrupt someone else, but who exactly those other individuals were in terms of their own simultaneous speech behavior. This relational view of behavior accords well with modern theories of leadership (e.g., Carter et al., 2015; Dansereau et al., 1995; Friedrich, Griffith, \& Mumford, 2016; Pearce \& Conger, 2002; Von Rueden \& Van Vugt, 2015) and social status 


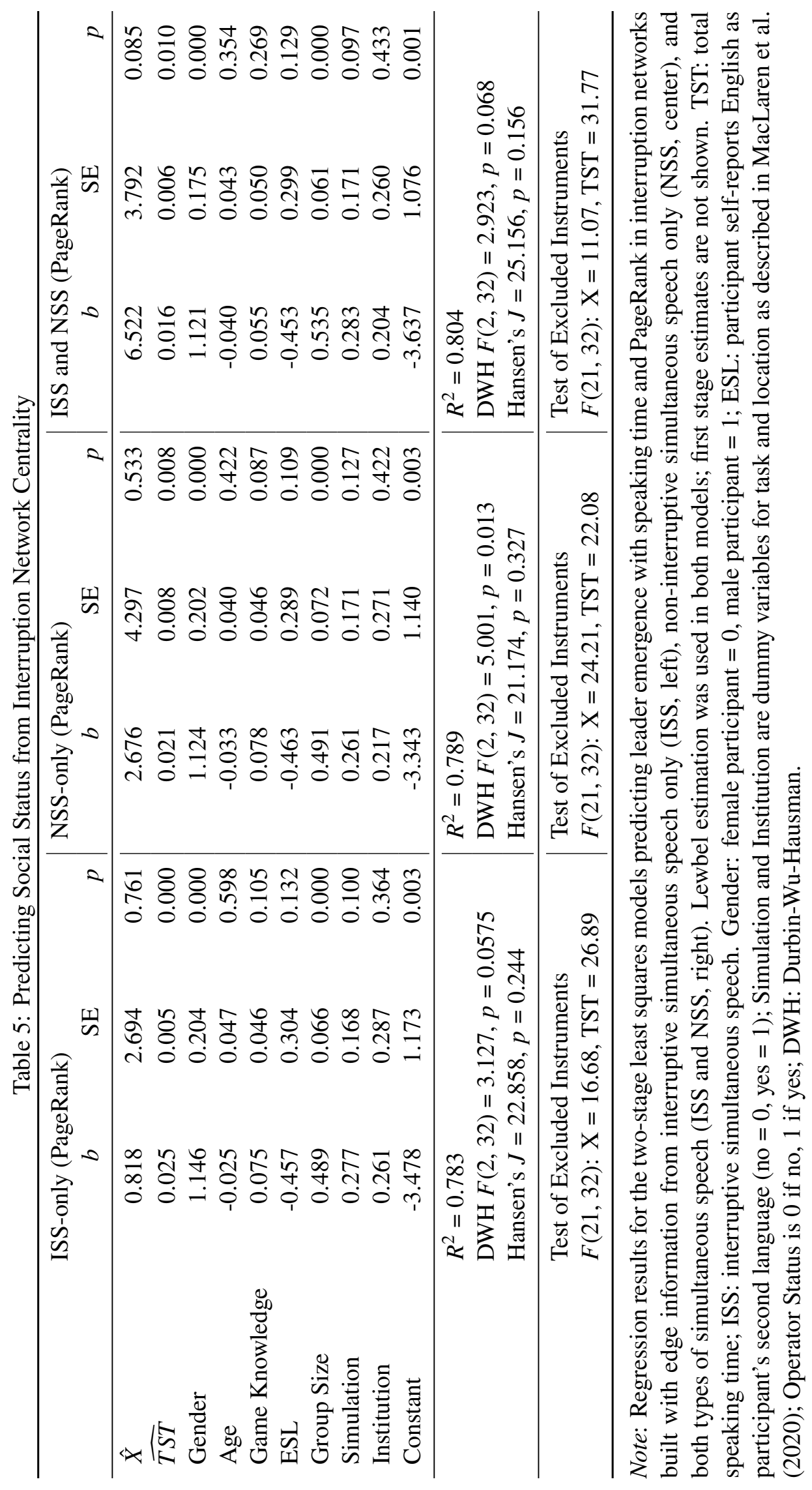


more generally (e.g., Anderson et al., 2015; Bendersky \& Pai, 2018).

More broadly, this study demonstrates the feasibility of behavioral networks - who behaved in what way towards whom-for leadership, management, and applied psychology research. Although proximity networks (Matusik et al., 2019) and constrained tie networks (Argote, Aven, \& Kush, 2018; Shore, Bernstein, \& Lazer, 2015) have been used, the interruption networks developed in this study join the the turn-based networks proposed by Sauer and Kauffeld (2013) to provide a new way of looking at behaviors and social structure in groups based on direct observations of discrete behaviors. We advocate a multilayer network approach in which different behaviors and attributions provide different information about the structure of relationships within a group.

The methods described in this study have advantages for other studies relying on unobtrusive measurements, such as those using sociometric badges in real organizations or in high fidelity testing environments. But there are also theoretical implications: the interruption network provides measures distinct from a "liking" construct (Yammarino et al., 2020) which point towards a group member who can be considered a leader. The network representation then allows for a non-linear, relational view of sociometric relationships, at least in the so-called vertical dimension, that may be used to test relational theories of leadership in observed behaviors. Outside of leadership, models of sociometric structure, particularly one such as this that can be sampled over time, can provide background information in other studies for which social structure may be a predictor or criterion: diversity, satisfaction, turnover intentions, and process loss may either influence or be influenced by social structure, and interruption networks could support assessments in these areas by providing an alternate, non-survey measure for assessing social structure.

\section{Summary of Findings}

Taken together, this investigation into simultaneous speech in informal, initially leaderless groups makes the following observations. First, in accordance with prior research, interruptions (ISS), without controlling for speaking time, positively predict leader emergence when controlling for endogeneity and a variety of individual and group level variables. Also in accordance with prior research, when controlling for speaking time the raw count of individual-level interruptions loses its incremental predictive validity. We recover the expected incremental validity by using centrality in networks of interruptions instead of the raw count, emphasizing the importance of patterns in behavioral relationships between individuals in a group. In short, our hypothesis appears to be supported in this data: patterns in simultaneous speech, as represented by interruption networks, do predict survey-assessed sociometric status.

Second, interruption networks appear to display patterns that indicate the importance of the direction and target of simultaneous speech behavior, which appears inconsistent with the idea that simultaneous speech is a random event predicated only on speaking time. Network motifs suggesting directed "flow", expected if interruption networks contain hierarchical mesoscale information, are found significantly more often than expected by chance; cyclic motifs that should appear equally often if directed "flow" is not important, are not. Furthermore, randomly changing the direction of interruption edges, which should have no effect if the direction of the empirically observed behavior is random, does have a 
substantial effect - at least at the sample level. Additional research is needed to ascertain whether the network level differences we observe in the data are simply due to the size and density of these networks or if they reveal important differences between social relationships in groups (Kivelä et al., 2014).

Third, exponential random graph analysis of interruption and turn-based reveal differential patterns in edge formation in these networks. For example, the operator role in this study, a facilitative role required by the task structure, appears central to the conversation, as seen in the turn-based networks, but does not seem associated with in-edges in interruption networks - consistent with the status interpretation of patterns in simultaneous speech. ERGM analysis also supports a variety of other ideas, from importance of speaking time alongside other conversational behaviors to the interpretation of simultaneous speech as a feature of language fluency. Gender is shown to have a complicated relationship to simultaneous speech, but in this data male gender appears inversely associated with out-edges in interruption networks. Men appear not to interrupt more than is expected based on their speaking time, but the data is consistent with the idea that women engage in more backchannel support for speakers than men do (Dabbs \& Ruback, 1987).

\section{Limitations}

There are several important limitations of this research. First is a problem of sample size. We made the decision to stick to published data: the sample size was already set. Although we have presented evidence that supports the importance of our primary finding, in a future study a larger sample size - either in terms of minutes of recording per group or number of groups - may be needed to more precisely estimate the relationship between simultaneous speech and social status while controlling for the confounding effects of speaking time.

Second, the social structure we model in this study is limited by the nonverbal behavior used to construct it. Interruptions are one of many possible nonverbal behaviors that can be associated with social status (Hall et al., 2005) or leadership (Gerpott et al., 2018): different behaviors may very well construct different models of social structure, which may in turn have different associations with gender. Additionally, this study did not assess other potential differences between male and female group members that may be relevant to leader emergence. Analysis of additional behaviors, as well as of the semantic content of the interruptions themselves, is planned for future studies.

Third, we have not considered the potential for different latent classes of simultaneous speech beyond the two (ISS and NSS) enumerated here. Recent research that indicates differences in attitudes towards interruptions based on similarity or dissimilarity of pitch and intensity between speakers, as well as the cultural and linguistic backgrounds of the speakers, plays an important role (Hilton, 2016). Future research is needed to further investigate the moderating — or mediating - role that other prosodic features have on the relationship between simultaneous speech and social status.

Fourth, this study validated interruption networks in ad hoc groups of student participants, constraining generalizability. However, the analysis presented here makes 
precise, quantitative predictions that can be tested against future data (Grahek, Schaller, \& Tackett, 2021; Yarkoni, 2019). For example, in a formal group, given a similar situation (e.g., a planning meeting), the highest interruption network PageRank value should correspond to the formal leader. If not, either that particular group may have an alternate informal hierarchy (DeRue \& Ashford, 2010) or, if there is a consistent difference across groups, it could indicate important boundary conditions on the interruption network representation: perhaps interruption networks, and the theories about sociometric relationships they encode, only apply to informal, zero-history groups. In either case, the interruption network provides a way to test these questions and this study provides benchmark data.

Fifth, we have argued for the construct validity of the interruption network, but we do not observe in this data whether individuals actually alter their behavior to align with the individual the interruption network method identifies as the leader. The interruption network procedure may have theoretical value, but group members' task behaviors are more in line with their attributions than with the nonverbal behaviors of others. Future work is needed to study a situation in which group member responses to co-member suggestions are tracked and compared to compliance or other sort of dependent action assessment to see if members act more in accordance with the behavior- or attribution-based group structure.

Finally, data collection and diarization procedures need to be improved to increase precision, accuracy, and robustness for field use. Specifically, automated diarization is preferred to reduce variability due to human coding. Despite the time consumed by coding these videos by hand, we have a limited data set with which to address many questions. Conclusions from this study may change with an increased number of observations (that is, an increased study sample size) or number of interactions (group tenure). Improved diarization techniques, some of which are under development, will further enhance the utility of the techniques descried above by enabling access to larger data sets and data recorded in a wider variety of study designs.

\section{Conclusion}

Modern conceptions of leadership heavily emphasize the relationships between individuals-it is not enough simply to know what one person is like, we must understand their connections with others. Alongside this theoretical development has been a consistent refrain concerning the importance of a leader's communication. In this study, we have demonstrated that communication behaviors that have been associated with attributions of leadership and social status can be modeled as a network in an informal group setting. Understanding who engages in what kind of simultaneous speech with whom may provide additional insight into who emerges as a leader in informal groups and can characterize complexities of within-group interaction that are not visible in speaking time alone. Network models based on nonverbal behaviors may provide a powerful way to connect studies of communication in groups with theories of leader emergence, leader behavior, and the structure of leadership ties.

\section{References}

Alvesson, M., \& Einola, K. (2019). Warning for excessive positivity: Authentic leadership 
and other traps in leadership studies. The Leadership Quarterly, 30(4), 383-395.

Anderson, C., Hildreth, J. A. D., \& Howland, L. (2015). Is the desire for status a fundamental human motive? A review of the empirical literature. Psychological Bulletin, 141(3), 574-601.

Antonakis, J., Bendahan, S., Jacquart, P., \& Lalive, R. (2010). On making causal claims: A review and recommendations. The Leadership Quarterly, 21(6), 1086-1120.

Argote, L., Aven, B. L., \& Kush, J. (2018). The effects of communication networks and turnover on transactive memory and group performance. Organization Science, 29(2), 191-206.

Banks, G. C., Gooty, J., Ross, R. L., Williams, C. E., \& Harrington, N. T. (2018). Construct redundancy in leader behaviors: A review and agenda for the future. The Leadership Quarterly, 29(1), 236-251.

Barrett, L., Henzi, S. P., \& Lusseau, D. (2012). Taking sociality seriously: the structure of multi-dimensional social networks as a source of information for individuals.

Philosophical Transactions of the Royal Society B: Biological Sciences, 367(1599), 2108-2118.

Bass, B. M. (2008). The Bass handbook of leadership: Theory, research, and managerial applications. Simon and Schuster.

Bavelas, A. (1950). Communication patterns in task-oriented groups. The Journal of the Acoustical Society of America, 22(6), 725-730.

Bavelas, A., Hastorf, A. H., Gross, A. E., \& Kite, W. R. (1965). Experiments on the alteration of group structure. Journal of Experimental Social Psychology, 1, 55-70.

Becker, J., Brackbill, D., \& Centola, D. (2017). Network dynamics of social influence in the wisdom of crowds. Proceedings of the National Academy of Sciences, 114(26), E5070-E5076.

Bendersky, C., \& Pai, J. (2018). Status dynamics. Annual Review of Organizational Psychology and Organizational Behavior, 5, 183-199.

Berger, J., Cohen, B. P., \& Zelditch, M. (1972). Status characteristics and social interaction. American Sociological Review, 37(3), 241-255.

Carter, D. R., DeChurch, L. A., Braun, M. T., \& Contractor, N. S. (2015). Social network approaches to leadership: An integrative conceptual review. Journal of Applied Psychology, 100(3), 597.

Cheng, J. T., Tracy, J. L., Foulsham, T., Kingstone, A., \& Henrich, J. (2013). Two ways to the top: Evidence that dominance and prestige are distinct yet viable avenues to social rank and influence. Journal of Personality and Social Psychology, 104(1), $103-125$.

Dabbs, J. M., \& Ruback, R. B. (1984). Vocal patterns in male and female groups. Personality and Social Psychology Bulletin, 10(4), 518-525.

Dabbs, J. M., \& Ruback, R. B. (1987). Dimensions of group process: Amount and structure of vocal interaction. In L. Berkowitz (Ed.), Advances in experimental social psychology (Vol. 20, pp. 123-169). Elsevier.

Dabbs, J. M., Ruback, R. B., \& Evans, M. S. (1987). "Grouptalk": Sound and silence in group conversation. In A. W. Siegman \& S. Feldstein (Eds.), Nonverbal behavior and communication (2nd ed., pp. 501-520). Erlbaum Hillsdale, NJ.

Dansereau, F., Yammarino, F. J., Markham, S. E., Alutto, J. A., Newman, J., Dumas, M., ... Keller, T. (1995). Individualized leadership: A new multiple-level approach. The Leadership Quarterly, 6(3), 413-450.

DeDeo, S., \& Hobson, E. A. (2021). From equality to hierarchy. Proceedings of the 
National Academy of Sciences, 118(21).

DeRue, D. S., \& Ashford, S. J. (2010). Who will lead and who will follow? a social process of leadership identity construction in organizations. Academy of Management Review, 35(4), 627-647.

Farley, S. D. (2008). Attaining status at the expense of likeability: Pilfering power through conversational interruption. Journal of Nonverbal Behavior, 32(4), 241-260.

Feldman, A., \& Gill, R. D. (2019). Power dynamics in supreme court oral arguments: The relationship between gender and justice-to-justice interruptions. Justice System Journal, 40(3), 173-195.

Feldstein, S., \& Welkowitz, J. (1987). A chronography of conversation: In defense of an objective approach. In A. W. Siegman \& S. Feldstein (Eds.), Nonverbal behavior and communication (2nd ed., pp. 435-499). Erlbaum Hillsdale, NJ.

Friedrich, T. L., Griffith, J. A., \& Mumford, M. D. (2016). Collective leadership behaviors: Evaluating the leader, team network, and problem situation characteristics that influence their use. The Leadership Quarterly, 27(2), 312-333.

Gerpott, F. H., Lehmann-Willenbrock, N., Silvis, J. D., \& Van Vugt, M. (2018). In the eye of the beholder? an eye-tracking experiment on emergent leadership in team interactions. The Leadership Quarterly, 29(4), 523-532.

Goodreau, S. M., Kitts, J. A., \& Morris, M. (2009). Birds of a feather, or friend of a friend? using exponential random graph models to investigate adolescent social networks. Demography, 46(1), 103-125.

Gorman, J. C., Grimm, D. A., Stevens, R. H., Galloway, T., Willemsen-Dunlap, A. M., \& Halpin, D. J. (2020). Measuring real-time team cognition during team training. Human Factors, 62(5), 825-860.

Grahek, I., Schaller, M., \& Tackett, J. L. (2021). Anatomy of a psychological theory: Integrating construct-validation and computational-modeling methods to advance theorizing. Perspectives on Psychological Science, 1-13.

Hall, J. A., Coats, E. J., \& LeBeau, L. S. (2005). Nonverbal behavior and the vertical dimension of social relations: a meta-analysis. Psychological Bulletin, 131(6), 898-924.

Hall, J. A., \& Friedman, G. B. (1999). Status, gender, and nonverbal behavior: A study of structured interactions between employees of a company. Personality and Social Psychology Bulletin, 25(9), 1082-1091.

Hall, J. A., Horgan, T. G., \& Murphy, N. A. (2019). Nonverbal communication. Annual Review of Psychology, 70, 271-294.

Heldner, M., \& Edlund, J. (2010). Pauses, gaps and overlaps in conversations. Journal of Phonetics, 38(4), 555-568.

Henrich, J., Chudek, M., \& Boyd, R. (2015). The big man mechanism: how prestige fosters cooperation and creates prosocial leaders. Philosophical Transactions of the Royal Society B: Biological Sciences, 370(1683), 20150013.

Hilton, K. (2016). The perception of overlapping speech: Effects of speaker prosody and listener attitudes. In Interspeech (pp. 1260-1264).

Holme, P., \& Saramäki, J. (2012). Temporal networks. Physics Reports, 519(3), 97-125.

Irish, J. T., \& Hall, J. A. (1995). Interruptive patterns in medical visits: the effects of role, status and gender. Social Science \& Medicine, 41(6), 873-881.

James, D., \& Clarke, S. (1993). Women, men, and interruptions: A critical review. In D. Tannen (Ed.), Gender and conversational interaction (pp. 231-280).

Kawakatsu, M., Chodrow, P. S., Eikmeier, N., \& Larremore, D. B. (2021). Emergence of 
hierarchy in networked endorsement dynamics. Proceedings of the National Academy of Sciences, 118(16).

Kivelä, M., Arenas, A., Barthelemy, M., Gleeson, J. P., Moreno, Y., \& Porter, M. A. (2014). Multilayer networks. Journal of Complex Networks, 2(3), 203-271.

Krackhardt, D. (1994). Graph theoretical dimensions of informal organizations. In K. M. Carley \& M. J. Prietula (Eds.), Computational organization theory (pp. 89-111). Psychology Press.

Krivitsky, P. N. (2012). Exponential-family random graph models for valued networks. Electronic Journal of Statistics, 6, 1100.

Lanaj, K., \& Hollenbeck, J. R. (2015). Leadership over-emergence in self-managing teams: The role of gender and countervailing biases. Academy of Management Journal, 58(5), 1476-1494.

Langville, A. N., \& Meyer, C. D. (2005). A survey of eigenvector methods for web information retrieval. SIAM Review, 47(1), 135-161.

Laskowski, K. (2010). Modeling norms of turn-taking in multi-party conversation. In Proceedings of the 48th annual meeting of the association for computational linguistics (pp. 999-1008).

Lewbel, A. (2012). Using heteroscedasticity to identify and estimate mismeasured and endogenous regressor models. Journal of Business \& Economic Statistics, 30(1), $67-80$.

MacLaren, N. G., Yammarino, F. J., Dionne, S. D., Sayama, H., Mumford, M. D., Connelly, S., . . others (2020). Testing the babble hypothesis: speaking time predicts leader emergence in small groups. The Leadership Quarterly, 31(5), 101409.

Martinko, M. J., Mackey, J. D., Moss, S. E., Harvey, P., McAllister, C. P., \& Brees, J. R. (2018). An exploration of the role of subordinate affect in leader evaluations. Journal of Applied Psychology, 103(7), 738-752.

Matusik, J. G., Heidl, R., Hollenbeck, J. R., Yu, A., Lee, H. W., \& Howe, M. (2019).

Wearable bluetooth sensors for capturing relational variables and temporal variability in relationships: A construct validation study. Journal of Applied Psychology, 104(3), 357-387.

Max Planck Institute for Psycholinguistics. (2018, August). ELAN (version 5.3) [Computer Software]. Nijmegen: Max Planck Institute for Psycholinguistics. Retrieved from https://tla.mpi.nl/tools/tla-tools/elan/

Milo, R., Shen-Orr, S., Itzkovitz, S., Kashtan, N., Chklovskii, D., \& Alon, U. (2002). Network motifs: simple building blocks of complex networks. Science, 298(5594), 824-827.

Morris, M., Handcock, M. S., \& Hunter, D. R. (2008). Specification of exponential-family random graph models: terms and computational aspects. Journal of Statistical Software, 24(4), 1548.

Ng, S. H., Brooke, M., \& Dunne, M. (1995). Interruption and influence in discussion groups. Journal of Language and Social Psychology, 14(4), 369-381.

Parker, A., Pallotti, F., \& Lomi, A. (2021). New network models for the analysis of social contagion in organizations: an introduction to autologistic actor attribute models. Organizational Research Methods, 10944281211005167.

Pearce, C. L., \& Conger, J. A. (2002). Shared leadership: Reframing the hows and whys of leadership. Sage Publications.

Reynolds, P. D. (1984). Leaders never quit: Talking, silence, and influence in interpersonal groups. Small Group Behavior, 15(3), 404-413. 
Riedl, C., Kim, Y. J., Gupta, P., Malone, T. W., \& Woolley, A. W. (2021). Quantifying collective intelligence in human groups. Proceedings of the National Academy of Sciences, 118(21).

Ruback, R. B., Dabbs, J. M., \& Hopper, C. H. (1984). The process of brainstorming: An analysis with individual and group vocal parameters. Journal of Personality and Social Psychology, 47(3), 558-567.

Sanchez-Cortes, D., Aran, O., Jayagopi, D. B., Schmid Mast, M., \& Gatica-Perez, D. (2013). Emergent leaders through looking and speaking: From audio-visual data to multimodal recognition. Journal on Multimodal User Interfaces, 7(1-2), 39-53.

Sauer, N. C., \& Kauffeld, S. (2013). Meetings as networks: Applying social network analysis to team interaction. Communication Methods and Measures, 7(1), 26-47.

Sayama, H., Cramer, C., Porter, M. A., Sheetz, L., \& Uzzo, S. (2016). What are essential concepts about networks? Journal of Complex Networks, 4(3), 457-474.

Schmid Mast, M. (2002). Dominance as expressed and inferred through speaking time. Human Communication Research, 28(3), 420-450.

Schmid Mast, M., \& Hall, J. A. (2004). Who is the boss and who is not? accuracy of judging status. Journal of Nonverbal Behavior, 28(3), 145-165.

Shore, J., Bernstein, E., \& Lazer, D. (2015). Facts and figuring: An experimental investigation of network structure and performance in information and solution spaces. Organization Science, 26(5), 1432-1446.

Smith-Lovin, L., \& Brody, C. (1989). Interruptions in group discussions: The effects of gender and group composition. American Sociological Review, 424-435.

Snijders, T. A., Van de Bunt, G. G., \& Steglich, C. E. (2010). Introduction to stochastic actor-based models for network dynamics. Social networks, 32(1), 44-60.

Stasser, G., Abele, S., \& Parsons, S. V. (2012). Information flow and influence in collective choice. Group Processes \& Intergroup Relations, 15(5), 619-635.

Tahir, Y., Yang, Z., Chakraborty, D., Thalmann, N., Thalmann, D., Maniam, Y., ... Dauwels, J. (2019). Non-verbal speech cues as objective measures for negative symptoms in patients with schizophrenia. PloS ONE, 14(4), e0214314.

Torrance, E. P. (1954, December). Some consequences of power differences on decisions in B-26 crews (Tech. Rep. No. AFPTRC-TR-54-128). Randolph Air Force Base, TX: Crew Research Laboratory, Air Force Personnel and Training Research Center, Air Research and Decelopment Command. (Approved by Roert L. French, Director, Crew Research Laboratory. Project No. 7713, Task No. 57157.)

Von Rueden, C., \& Van Vugt, M. (2015). Leadership in small-scale societies: Some implications for theory, research, and practice. The Leadership Quarterly, 26(6), 978-990.

Ward, N. G. (2019). Prosodic patterns in English conversation. Cambridge University Press.

Wasserman, S., \& Faust, K. (1994). Social network analysis: Methods and applications. Cambridge University Press.

Wasserstein, R. L., \& Lazar, N. A. (2016). The ASA statement on $p$-values: Context, process, and purpose. The American Statistician, 70(2), 129-133.

Wooldridge, J. M. (2010). Econometric analysis of cross section and panel data. MIT Press.

Yammarino, F. J., Cheong, M., Kim, J., \& Tsai, C.-Y. (2020). Is leadership more than "i like my boss"? Research in Personnel and Human Resources Management, 38.

Yammarino, F. J., \& Gooty, J. (2019). Cross-level models. In S. E. Humphrey \& 
J. M. LeBreton (Eds.), The handbook of multilevel theory, measurement, and analysis (pp. 563-585). American Psychological Association.

Yarkoni, T. (2019). The generalizability crisis. Preprint. PsyArXiv. https://doi. org/10.31234/osf. io/jqw35.

Zaccaro, S. J., Green, J. P., Dubrow, S., \& Kolze, M. (2018). Leader individual differences, situational parameters, and leadership outcomes: A comprehensive review and integration. The Leadership Quarterly, 29(1), 2-43. 